\title{
Dependencia al ejercicio físico e indicadores del estado de ánimo en deportistas universitarios
}

\section{Exercise dependence and mood states indicators in university athletes}

\author{
Dependência ao exercício físico e indicadores do estado \\ de ânimo em universitários esportistas
}

\author{
Cristina Reche García ${ }^{1}$, Alejandro Martínez-Rodríguez ${ }^{2}$ y Francisco J. Ortín Montero ${ }^{3}$
}

\begin{abstract}
${ }^{1}$ Departamento de Ciencias de la Salud - Facultad de Enfermería - UCAM Universidad Católica San Antonio de Murcia - España. ${ }^{2}$ Departamento de Ciencias de la Actividad Física y del Deporte - Facultad de Deporte - UCAM Universidad Católica San Antonio de Murcia - España. y ${ }^{3}$ Universidad de Murcia - España.
\end{abstract}

Resumen: El objetivo de este estudio fue conocer la prevalencia de la dependencia al ejercicio físico en deportistas universitarios; analizar las diferencias en función del sexo, modalidad deportiva y tiempo de entrenamiento. Además, analizar su relación y sensibilidad con los estados de ánimo. Participaron 255 estudiantes ( 212 hombres y 43 mujeres) con edades comprendidas entre los 18 y 43 años $(M=21,25 ; \mathrm{DT}=4,28)$. Se administró la versión espańola de la "Escala de Dependencia del Ejercicio- Revisada" (EDS-R; Sicilia y González, 2011) y el Profile of Mood States (POMS; McNair, Lorr y Droppleman, 1971). Los resultados mostraron una dependencia al ejercicio físico representada en un $6 \%$ de los participantes del estudio, sin diferencias estadísticamente significativas en función del sexo o modalidad deportiva. En cuanto al tiempo de entrenamiento sí aparecieron diferencias, siendo los que presentaban alta dedicación los que presentaban mayores síntomas de dependencia al ejercicio físico, frente a los que presentaban una dedicación saludable. La dependencia al ejercicio se relacionó con un perfil del estado de ánimo de tensión, depresión, cólera, fatiga y debilidad. Los resultados han permitido identificar características del proceso de dependencia del ejercicio e indicadores psicológicos que permiten detectar signos del trastorno, mediante la evaluación psicológica durante los períodos de entrenamiento, para prevenir el desarrollo completo del problema.

Palabras clave: Adicción al ejercicio, indicadores psicológicos, estudiantes, prevalencia

Abstract: The aim of this study was to determine exercise dependence prevalence in college athletes; analyze differences by gender, kind of sport and training time. In addition, to analyze its relationship with sensitivity and moods. Involved 255 students (212 men and 43 women) aged between 18 and 43 years $(M=21.25, S D=4.28)$. Spanish version of the Exercise Dependence Scale -Revised (EDS- R; Sicilia and González, 2011) and Profile of Mood States (POMS, McNair, Lorr and Droppleman, 1971) were administered. The results showed a dependence on exercise represented $6 \%$ of the study participants, no statistically significant differences by gender or sport. Regarding the training time differences did appear, being those with high dedication which had higher symptoms of exercise dependence, compared to those with a healthy dedication. Exercise dependence was associated with a profile mood of tension, depression, anger, fatigue and weakness. The results have identified characteristics of the process of exercise and psychological dependence indicators for signs of the disorder through psychological evaluation during training periods to prevent the full development of the problem.

Keywords: Exercise addiction, psychological indicators, students, prevalence.

Resumo: O objetivo deste estudo foi conhecer a prevalência da dependência ao exercício físico em universitários esportistas; analisar as diferenças por gênero, modalidade desportiva e período de treinamento. Além disso, analisar a relaçấo e sensibilidade com os estados de ânimo. Participaram 255 estudantes (212 homens e 43 mulheres) com idades entre 18 e 43 anos $(M=$ 21,25; DT = 4,28). Administrou-se a versão espanhola da "Escala de Dependência do Exercício- Revisada” (EDS-R; Sicilia e González, 2011) e o Profile of Mood States (POMS; McNair, Lorr e Droppleman, 1971). Os resultados mostraram uma dependência ao exercício físico, representada por $6 \%$ dos participantes do estudo, sem diferenças estatisticamente significativas entre gênero ou modalidade esportiva. Houve diferenças enquanto ao período de treinamento, sendo que, os sujeitos que apresentavam maior dedicação apresentavam altos sintomas de dependência ao exercício físico, comparando aos que se dedicavam de maneira saudável. A dependência ao exercício foi relacionada com o perfil de estado de ânimo de tensão, depressão, cólera, fadiga e debilidade. Os resultados permitiram esclarecer quais as características do processo de dependência do exercício e indicadores psicológicos que permitem detectar signos de transtorno, mediante a valoração psicológica durante os treinamentos, para prevenir o desenvolvimento do problema.

Palavras chave: Vício ao exercício, indicadores psicológicos, estudantes, prevalência.

\section{Introduccion}

Son escasos los trabajos destinados al estudio y análisis de las características que presentan los procesos de dependencia Dirección para correspondencia [Correspondence address]: Cristina
Reche García. Facultad de Enfermería. Universidad Católica de Murcia.
Campus de Los Jerónimos, s/n, 30107, Guadalupe, Murcia (España). E-mail: creche@ucam.edu al deporte. La prevalencia de adicción al deporte, así como su formación y curso, no ha sido completamente estudiada hasta el momento (Krivoschelov y Lushnikov, 2011), y el concepto de dependencia física y/o psicológica del ejercicio físico necesita de una mayor clarificación y estudio (Kjelsas, Augestad, y Götestam, 2003); ya que no existe consenso nosológico, 
ni se reconoce como un trastorno conductual independiente (Remor, 2005). Sin embargo, la dependencia al ejercicio físico puede ser categorizada como una adicción comportamental (Demetrovics y Kurimay, 2008), para la cual hay varias explicaciones plausibles (Adams y Kirkby, 2002).

La evidencia sugiere que la actividad física regular está asociada a la mejora de estados psicológicos, así como a una posible adicción o dependencia (Christie y Chesher, 1982), que causaría cambios en el estado de ánimo con su deprivación (Mondin et al., 1996).

Entre otras alteraciones, la alteración psicológica se manifiesta en los deportistas cuando los parámetros de carga y volumen son muy elevados, ya que los sujetos, en muchas ocasiones no son capaces de asimilarlos correctamente, manifestándose incluso signos y síntomas evidentes de sobreentrenamiento (Molinero, Salguero y Márquez, 2011).

En este punto, el bienestar psicológico asociado a la práctica regular de ejercicio físico pasa a convertirse en un problema psicosocial y sanitario (Jiménez, Martínez, Miró, y Sánchez, 2008), manifestándose en el individuo un patrón desadaptativo con un deterioro o malestar clínicamente significativos, con consecuencias adversas y recurrentes que alterarían el estado afectivo de los deportistas (Antunes, Stela, Santos, Bueno, y De Mello, 2005; Antunes, Andersen, Tufik, y De Mello, 2006; Rosa, De Mello, Negrao, y Souza-formigoni, 2004) (en Modolo, Mello, Gimenez, Tufik, y Antunes, 2009), con manifestaciones de tensión, fatiga y depresión; factores psicológicos que junto a factores físicos, comportamentales, socioculturales, y del tipo de ejercicio, se asocian a la dependencia al ejercicio físico (Adams, Miller, y Kraus, 2003).

Es conocido que algunos aspectos negativos propios de una adicción o dependencia (Kjelsas et al., 2003) que pudiera provocar el ejercicio excesivo (Allegre, Souvulle, Therme, y Griffiths, 2006), son la aparición de síntomas de abstinencia tales como irritabilidad, ansiedad y depresión; insomnio, tensión y/o somatizaciones (Morgan, 1979; Morris, Steinberg, Sykes, y Salmon, 1990) (en Caracuel y Arbinaga, 2010). Además, otros síntomas físicos como la tolerancia (Hausenblas y Symons, 2002a). Sin embargo, estudios recientes no relacionan deterioros en el estado de ánimo con una elevada dedicación al entrenamiento (Guszkowska y Rudnicki, 2012), ni con el trastorno por dependencia al ejercicio (utilizando la escala EDE) (Antunes et al., 2006).

En cuanto a las diferencias por género, encontramos estudios que refieren niveles más elevados de dependencia al ejercicio físico en mujeres frente a hombres (e.g., Masters y Lambert, 1989; Pierce et al., 1997; Summers et al., 1983) (en Allegre, Pierre, y Griffiths, 2007), sin embargo otros no encuentran diferencias (e.g., Furst y Germone, 1993) (en Allegre et al, 2007). Recientemente y con la aplicación del cuestionario EDS, se concluye que es en hombres dónde aparecen puntuaciones más elevadas en dependencia al ejercicio físico, y que otro resultado pudiera deberse a la utilización de otros instrumentos de evaluación que valoren diferentes criterios (Weik y Hale, 2009); utilizando el EDS-R aparece la misma conclusión, exceptuando el factor abstinencia (González-Cutre y Sicilia, 2013).

En relación a las modalidades deportivas individuales y colectivas, la evidencia científica refiere mejor estado de ánimo en las colectivas, presentando niveles similares de dependencia (Modolo et al., 2009).

Para evaluar la adicción al ejercicio físico se han utilizado cuestionarios que facilitan una evaluación de la adicción al ejercicio físico de forma específica y adaptada. Su evolución cronológica, siguiendo con propiedades psicométricas fundamentadas, ha sido la siguiente: Exercise Dependence Questionnaire, EDQ (Ogden, Veale, y Summers, 1997) basado en criterios de dependencia al ejercicio físico (Veale, 1987; Veale, 1995); Exercise Dependence Scale, EDS y EDS-R (Hausenblas y Symons, 2002b); Exercise Addiction Inventory, EAI (Terry, Szabo, y Griffiths, 2004); la Escala de Dependência de Exercício, EDE (Rosa, Mello, y Souza-Formigoni, 2003); y la versión española de Exercise Dependence Scale-Revised, EDS-R (Sicilia y González, 2011).

Se requiere más investigación para conocer los factores precipitantes y perpetuantes asociados a la dependencia al ejercicio (Hausenblas \& Symons, 2002a), y se considera oportuno realizar estudios de carácter epidemiológico que permitan conocer la incidencia y prevalencia del fenómeno (Arbinaga, 2004); así como estudios que identifiquen síntomas biopsicosociales en distintas poblaciones, correlatos de adaptación, etiología, mantenimiento y remisión de síntomas (Adams et al., 2003).

Este estudio tiene por objeto conocer la prevalencia de la dependencia al ejercicio físico en deportistas universitarios y analizar su relación y sensibilidad con el estado de ánimo en la práctica deportiva. Además, analizar las diferencias en dependencia del ejercicio en función del sexo, modalidad deportiva, y tiempo de entrenamiento. Así, se podrán establecer estrategias o consideraciones que permitan la detección e intervención del psicólogo deportivo en el ámbito competitivo y fuera de él, asegurando la continuidad del ejercicio físico sin los aspectos negativos propios de una adicción o dependencia. Es una forma de contar con indicadores que permitan detectar e identificar signos del trastorno, mediante la evaluación psicológica durante los períodos de entrenamiento, para prevenir el desarrollo completo del problema.

\section{Método}

\section{Participantes}

La muestra está formada por 255 estudiantes universitarios (43 mujeres y 212 hombres) con edades comprendidas entre los 18 y los 43 años $(M=21,25 ; \mathrm{DT}=4,28)$ y en periodo de 
entrenamiento; practicantes de distintas modalidades deportivas de ámbito universitario (el $49 \%$ participaban en modalidades colectivas de equipo y $51 \%$ en deportes individuales). El 98\% practicaban ejercicio físico como mínimo 1 hora semanal y hasta 4 horas y el $2 \%$ restante más de 4 horas y hasta un máximo de 10 .

\section{Instrumentos}

La Escala Revisada de Dependencia del Ejercicio (EDS-R; Sicilia \& González, 2011), evalúa el riesgo de dependencia de deportistas. Consta de 21 ítems, que hacen referencia a creencias y comportamientos que han ocurrido en los últimos 3 meses. Los sujetos responden en una escala tipo Lickert de 1 a 6 ( 0 =nunca; $6=$ siempre) a una serie de afirmaciones.

Se escoge esta prueba, tras una exhaustiva revisión de instrumentos, por su actualización, validación y versión en espańol. Esta escala conceptualiza la dependencia al ejercicio físico basándose en los criterios de dependencia a sustancias del DSM-IV (American Psychiatric Association, 1994): Tolerancia: Definida como la necesidad de aumentar la cantidad de ejercicio para conseguir el efecto deseado, o la disminución del efecto con el uso continuado de la misma cantidad de ejercicio; Abstinencia: Manifestada por cualquier síntoma característico de abstinencia del ejercicio (e.g., ansiedad, fatiga, agotamiento), o la realización de ejercicio para evitar los síntomas de abstinencia; Efectos deseados: El ejercicio es a menudo realizado durante más tiempo o en mayor cantidad de lo que inicialmente se pretendía; Falta de control: Existe un deseo persistente o un esfuerzo infructuoso de controlar o interrumpir la realización de ejercicio; Tiempo: Se emplea mucho tiempo en actividades relacionadas con el ejercicio; Reducción de otras actividades: Reducción de importantes actividades sociales, laborales y recreativas; Continuación: Se continúa realizando ejercicio a pesar de tener conciencia de problemas psicológicos o físicos persistentes, probablemente causados o empeorados por la realización de ejercicio (e.g., continuar corriendo a pesar de una lesión).

El Profile of Mood States (POMS; McNair, Lorr y Droppleman, 1971), en su forma abreviada de 29 ítems y versión en castellano (Fuentes, Balaguer, Meliá y García-Merita, 1995) es eficaz en el diagnóstico de estados alterados por el entrenamiento intensivo.

Sin duda, el POMS es el instrumento más utilizado para registrar los estados de ánimo en contextos de entrenamiento, y el que se cita con mayor frecuencia en la literatura científica. El POMS es un autoinforme que contiene una lista de adjetivos a partir de los que se obtiene una evaluación global de las alteraciones del estado de ánimo, y 5 escalas: Depresión, Vigor, Cólera, Tensión y Fatiga. Los sujetos responden en una escala de 0 a 4 ( 0 =nada; $4=$ muchísimo) valorando los adjetivos presentados a la pregunta: “ ¿Cómo te has sentido durante la semana pasada, incluyendo el día de hoy?”; o bien “¿cómo te has sentido durante el día de hoy?” o “¡cómo te sientes ahora mismo?".

\section{Procedimiento}

Para la recogida de datos y aplicación de los cuestionarios, se solicitó participación voluntaria entre los estudiantes universitarios de primer curso del Grado de Ciencias de la Actividad Física y del Deporte 2012/2013 de la Universidad Miguel Hernández y de la Universidad de Alicante. De esta forma, se reunieron a todos los colaboradores, se les explicó y describió la investigación y la forma de cumplimentación de la prueba psicológica pidiéndoles su colaboración para el reparto entre los participantes. Se instó a que consultasen cualquier tipo de duda que les pudiese surgir y que devolviesen al colaborador los cuestionarios antes de retirarse de las instalaciones deportivas.

Todos los participantes cumplimentaron la prueba de forma individual a lo largo de una sesión y firmaron su consentimiento informado. Un criterio de exclusión fueron las pruebas con falta de cumplimentación de datos esenciales y/o ítems necesarios. El procedimiento fue aprobado por el Comité de Ética de la Universidad Católica de Murcia.

\section{Análisis de los datos}

Para la descripción de la muestra, y la prevalencia de la dependencia al ejercicio físico, se realizaron análisis descriptivos. Seguidamente, para analizar las diferencias en dependencia del ejercicio físico en función del sexo, el tipo de actividad deportiva y el tiempo de entrenamiento; se comprobó que las puntuaciones obtenidas cumplían los criterios de normalidad, independencia y homogeneidad de varianzas. El análisis de las diferencias ese llevó a cabo a partir de estadísticos de contraste de comparación de medias (pruebas t de Student para muestras independientes).

Para la evaluación de las relaciones entre los estados de ánimo y la dependencia al ejercicio físico se realizaron correlaciones entre el EDS-R y las escalas que componen el POMS: Depresión, Vigor, Cólera, Tensión y Fatiga; así como con el POMS total.

Para efectuar los análisis estadísticos se utilizó el programa SPSS en su versión 21.0. A efectos de interpretación y análisis de los datos el nivel de confianza asumido fue de 0,05 $(p<, 05)$.

\section{Resultados}

A continuación se presentan los análisis descriptivos, que hacen referencia a la dependencia al ejercicio físico de los participantes, donde el $6 \%$ presenta dependencia al ejercicio físico, y el $94 \%$ restante no la presenta. Diferenciando en función del sexo encontramos que las mujeres son dependientes en un $9 \%$ de los 
casos, frente a los hombres que lo son en un 6\%; los participantes en deportes de equipo un $5 \%$ frente al $8 \%$ de los deportistas individuales; y los que entrenan 4 o menos horas un 6\%, frente al 25\% que lo hacen con un alta dedicación (Tabla 1).

Tabla 1. Descriptivos de prevalencia de la dependencia al ejercicio físico diferenciando por sexo, modalidad deportiva y horas de entrenamiento semanales, expresados en número de sujetos y porcentaje dentro de la población (n, \%).

\begin{tabular}{|c|c|c|c|c|}
\hline & & Dependencia & No dependencia & Total \\
\hline \multirow{2}{*}{ Sexo } & Mujer & $49 \%$ & $3991 \%$ & $4317 \%$ \\
\hline & Hombre & $126 \%$ & $20094 \%$ & $21283 \%$ \\
\hline Total & & $166 \%$ & $23994 \%$ & $255100 \%$ \\
\hline \multirow{2}{*}{ Modalidad deportiva } & Equipo & $65 \%$ & $12195 \%$ & $12749 \%$ \\
\hline & Individual & $108 \%$ & $11892 \%$ & $12851 \%$ \\
\hline Total & & $166 \%$ & $23994 \%$ & $255100 \%$ \\
\hline \multirow{2}{*}{ Horas de entreno } & $\leq 4$ & $156 \%$ & $23694 \%$ & $25198 \%$ \\
\hline & $>4$ & $125 \%$ & $375 \%$ & $42 \%$ \\
\hline Total & & $166 \%$ & $23994 \%$ & $255100 \%$ \\
\hline
\end{tabular}

Para conocer si existen diferencias significativas en dependencia en función del sexo de los participantes, de la modalidad deportiva y de las horas de entrenamiento se realizaron pruebas t de Student (Tabla 2). De los resultados se extrae que no existen diferencias estadísticamente significativas $\left({ }^{*} p<, 05\right)$ en dependencia en función del sexo del deportista, ni en función de la modalidad deportiva. Sin embargo, encontramos diferencias en dependencia al ejercicio físico en función de las horas de entrenamiento con un tamaño del efecto medio $(p=, 00 ; d=-, 41)$.

Tabla 2. Estadística descriptiva e inferencial de la dependencia al ejercicio físico en función del sexo, modalidad deportiva y horas de entrenamiento.

\begin{tabular}{|c|c|c|c|c|c|c|c|}
\hline & & $\mathrm{N}$ & Media & Dt & $d$ & $t$ & $p$ \\
\hline \multirow{2}{*}{ Sexo } & Mujer & 43 & 59,44 & 17,80 & \multirow[t]{2}{*}{,- 19} & \multirow[t]{2}{*}{$-1,20$} & \multirow[t]{2}{*}{, 23} \\
\hline & Hombre & 212 & 62,62 & 15,43 & & & \\
\hline \multirow{2}{*}{ Modalidad deportiva } & Equipo & 127 & 62,85 & 15,24 & \multirow[t]{2}{*}{, 09} & \multirow[t]{2}{*}{0,76} & \multirow[t]{2}{*}{,44 } \\
\hline & Individual & 128 & 61,33 & 16,49 & & & \\
\hline \multirow{2}{*}{ Horas de entrenamiento a la semana } & Saludable & 168 & 59,90 & 16,30 & \multirow[t]{2}{*}{,- 41} & \multirow[t]{2}{*}{$-3,10$} & \multirow[t]{2}{*}{, 00 } \\
\hline & Alta dedicación & 87 & 66,31 & 14,15 & & & \\
\hline
\end{tabular}
${ }^{*} p<.05$

Las correlaciones entre las dimensiones del POMS y la depen- dencia al ejercicio físico figuran en la Tabla 3.

Tabla 3. Correlaciones entre el EDS-R y las dimensiones del POM-S.

\begin{tabular}{lccccccc}
\hline & EDS-R & Tensión & Depresión & Cólera & Fatiga & Vigor & POMS \\
\hline EDS-R & 1 &, $21^{* *}$ &, $14^{*}$ &, $25^{* *}$ &, $17^{* *}$ & $-.21^{* *}$ & $.19^{* *}$ \\
Tensión &, $21^{* *}$ & 1 &, $46^{* *}$ &, $54^{* *}$ &, $30^{* *}$ & .00 & $.71^{* *}$ \\
Depresión &, $14^{*}$ &, $46^{* *}$ & 1 &, $71^{* *}$ &, $42^{* *}$ & $.21^{* *}$ & $.81^{* *}$ \\
Cólera &, $25^{* *}$ &, $54^{* *}$ &, $71^{* *}$ & 1 &, $39^{* *}$ & .06 & $.83^{* *}$ \\
Fatiga &, $17^{* *}$ &, $30^{* *}$ &, $42^{* *}$ &, $39^{* *}$ & 1 & .11 & $.65^{* *}$ \\
Vigor- &,$- 21^{* *}$ &, 00 &, $21^{* *}$ &, 06 &, 11 &, $35^{* *}$ \\
POMS &, $19^{* *}$ &, $71^{* *}$ &, $81^{* *}$ &, $83^{* *}$ &, $65^{* *}$ &, $35^{* *}$ & $.35^{*}$ \\
\hline
\end{tabular}

**. La correlación es significativa al nivel 0,01 (bilateral).

*. La correlación es significante al nivel 0,05 (bilateral). 
Los resultados sugieren que el EDS-R y las dimensiones del POMS (tensión, depresión, cólera, fatiga y vigor) mantienen relaciones entre sí, siendo interdependientes.

\section{Discusión}

El fenómeno de la dependencia al ejercicio físico presenta una baja prevalencia entre los participantes de nuestro estudio, no existiendo diferencias entre hombres y mujeres en la manifestación del mismo; en la misma línea que estudios referidos por Allegre et al. (2007) y en contraposición con otros que señalan lo contrario, exceptuando alguna sintomatología (González-Cutre y Sicilia, 2013) o utilizando una escala diferente a la seleccionada para la evaluación de la dependencia (Weik y Hale, 2009).

Tampoco encontramos diferencias en dependencia al ejercicio físico de deportistas universitarios por las características de su deporte, si es individual o colectivo, resultado afín a Modolo et al. (2009).

Los deportistas que practican ejercicio más de 4 horas a la semana, con una alta dedicación, por encima de las recomendaciones mínimas saludables (Garber et al., 2011); presentan una media global más elevada de los síntomas consistentes con una dependencia al ejercicio físico, que los que practican menos horas. Manifestando el empleo de una cantidad de tiempo significativamente más elevado en actividades relacionadas con el ejercicio físico, uno de los síntomas característicos de los trastornos por dependencia. Cuestión que se encuentra en la misma línea que estudios recientes (Reche y Gómez, 2014) y en divergencia con otros que señalan una dependencia al ejercicio físico relacionada con la participación elevada del tiempo en el deporte, y no tanto con su duración y frecuencia (Guszkowska y Rudnicki, 2012).

Siguiendo a Modolo et al. (2009) la dependencia de estos deportistas se ha visto relacionada con alteraciones en los estados afectivos. En concreto, con aumento de la tensión, depresión, cólera, fatiga y disminución del vigor; en la misma línea que investigaciones de Caracuel y Arbinaga (2010), que refieren relaciones con irritabilidad, ansiedad y depresión; insomnio, tensión y/o somatizaciones; y que estudios de Adams et al. (2003), que relacionan la dependencia al ejercicio físico con tensión, fatiga y depresión. Sin embargo, no se encuen- tran en consonancia con Antunes et al. (2006); que señalan no encontrar relaciones utilizando una escala diferente que valora diferentes criterios de dependencia a los utilizados en el EDS-R.

Se han identificado como síntomas o indicadores psicológicos de la dependencia al ejercicio físico en deportistas universitarios en periodo de entrenamiento, la tensión, depresión, cólera, fatiga y debilidad. Esto nos permite reconocer los signos del trastorno, para prevenir el desarrollo completo del problema, evitar lesiones asociadas al mismo (Tello, Jouvion, Boulard, Marimoutou, Cazenave, y Théfenne, 2012), y otras alteraciones psicológicas como trastornos de la imagen y de alimentación (Demetrovics y Kurimay, 2008).

Asimismo, el número de lesiones aumenta conforme lo hacen las horas de práctica deportiva, además de estar relacionadas con los aspectos psicológicos del deportista, existe una influencia de la lesión en el estado de ánimo, que pueden desencadenar limitaciones del rendimiento deportivo (Olmedilla, Ortega y Gómez, 2014)

En cuanto a las limitaciones de estudio encontradas decir que parece recomendable combinar la evaluación psicológica con la determinación de algunas variables biológicas, entre las que cabe destacar el cortisol. Además, realizar estudios que detecten la dependencia al ejercicio físico diferenciando entre duración, frecuencia, intensidad, y práctica en el tiempo del mismo.

\section{Aplicaciones prácticas}

El entendimiento de porqué una persona que desea realizar ejercicio físico alcanza niveles desadaptativos hasta el punto de depender de ello, se postula como un conocimiento cada vez más necesario y al mismo tiempo común. Conocer el estado emocional de los mismos, las necesidades que le llevan a entender el deporte como un único estilo de vida, a hipotecar sus esfuerzos y compromisos deportivos por encima de los personales o laborales conlleva la aparición de problemas adaptativos y de relación. Los deportistas que realizan ejercicio como un complemento de su estilo de vida, deben reconocer y entenderlo como tal y nunca ofrecer señales que les permitan entrar en complejos estados emocionales de difícil manejo o resolución.

\section{Referencias}

1. Adams, J. M., Miller, T. W., \& Kraus, R. F. (2003). Exercise dependence: diagnostic and therapeutic issues for patients in psychotherapy. Journal of Contemporary Psychotherapy, 33(2), 93-107.

2. Adams, J., \& Kirkby, R. J. (2002). Excessive exercise as an addiction: a review. Addiction Research and Theory, 10, 415-37.

3. Allegre, B., Souvulle, M., Therme, P., \& Griffiths, M. D. (2006). Definitions and measures of exercise dependence. Addiction Research and Theory, 14, 631-46.

4. Allegre, B., Therme, P., \& Griffiths, M. (2007). Individual factors and the context of physical activity in exercise dependence: A prospective study of 'ultra-marathoners'. International Journal of Mental Health and Addiction, 5(3), 233-243.

5. American Psychiatric Association (APA). (1994). Diagnostic and statistical manual of mental disorders (4th ed). Washington, DC: Author.

6. Antunes, H. K. M., Stella, S. G., Santos, R. F., Bueno, O. F. A., \& Mello, M. T. D. (2005). Depression, anxiety and quality of life scores in seniors after an endurance exercise program. Revista Brasileira de Psiquiatria, 27(4), 266-271. 
7. Antunes, H. K. M., Andersen, M. L., Tufik, S., \& De Mello, M. T. (2006). O estresse físico ea dependência de exercício físico. Revista Brasileira de Medicina do Esporte, 12(5), 234-238.

8. Arbinaga, F. (2004). Dependencia del ejercicio. Cuadernos de Medicina Psicosomática y Psiquiatría de Enlace, 71(72), 24-32.

9. Caracuel, E. \& Arbinaga, I. (2010). Repercusión del ejercicio físico sobre la salud. Apuntes de Psicología, 28(2), 315-328.

10. Christie, M. J., \& Chesher, G. B. (1982). Physical dependence on physiologically released endogenous opiates. Life sciences, 30(14), 11731177.

11. Demetrovics, Z. \& Kurimay, T. (2008). Exercise addiction: a literature review. Psychiatria Hungarica, 23 (2), 129-41.

12. Fuentes, I., Balaguer, I., Meliá, J. L. \& García-Merita, M. L. (1995). Forma abreviada del Perfil de los Estados de Ánimo (POMS). Actas del V Congreso Nacional de Psicología de la Actividad Física y el Deporte, (pp. 29-39). Valencia. Universitat de València.

13. Garber, C. E., Blissmer, B., Deschenes, M. R., Franklin, B. A., Lamonte, M. J., Lee, I. M., ... \& Swain, D. P. (2011). American College of Sports Medicine position stand. Quantity and quality of exercise for developing and maintaining cardiorespiratory, musculoskeletal, and neuromotor fitness in apparently healthy adults: guidance for prescribing exercise. Medicine and Science in Sports and Exercise, 43(7), 13341359.

14. González-Cutre, D. \& Sicilia, A. (2012). Dependencia del ejercicio físico en usuarios españoles de centros de acondicionamiento físico (fitness): diferencias según el sexo, la edad y las actividades practicadas. Behavioral Psychology/Psicología Conductual, 20(2), 349-364.

15. Guszkowska, M., \& Rudnicki, P. (2012). Mood changes in physically active men in situations of imagined discontinuation of physical exercises as an indication of the risk of addiction to physical exercises. Polish Journal of Sport and Tourism, 19(1), 16-20.

16. Hausenblas, H. A., \& Symons, D. (2002a). Exercise dependence: A systematic review. Psychology of Sport and Exercise, 3, 89-123. doi:10.1016/1469-0292(00)00015-7

17. Hausenblas, H. A., \& Symons, D. (2002b). Exercise Dependence Scale-21 Manual. Recuperado de http://www.personal.psu.edu/dsd11/ EDS/EDS21Manual.pdf

18. Jiménez, M. G., Martínez, P., Miró, E., \& Sánchez, A. I. (2008). Bienestar psicológico y hábitos saludables: ¿están asociados a la práctica de ejercicio físico? International Journal of Clinical and Health Psychology, 8(1), 185-202.

19. Kjelsas, E., Augestad, L. B., \& Götestam, K.G. (2003). Exercise dependence in physically activa women. The European Journal of Psychiatry, 17, 145-155.

20. Krivoschekov, S. G., \& Lushnikov, O. N. (2011). Psycho physiology of sports addictions (Exercise Addiction). Human Physiology, 37 (4), 509-13.

21. Mcnair, D. M., Lorr, M. \& Droppleman, L. F. (1971). Manual for the profile of mood states. San Diego, CA: Educational and Industrial Testing Service.
22. Modolo, V. B., Mello, M. T. D., Gimenez, P. R. B. D., Tufik, S., \& Antunes, H. K. M. (2009). Dependência de exercício físico: humor, qualidade de vida em atletas amadores e profissionais; Physical exercise dependence: mood, quality of life in amateur and professional athletes. Revista Brasileira de Medicina do Esporte, 15(5), 355-359.

23. Molinero, O., Salguero, A., \& Márquez, S. (2011). Análisis de la recuperación-estrés en deportistas y relación con los estados de ánimo: un estudio descriptivo. Cuadernos de Psicología del Deporte, 11 (2), 47-55.

24. Mondin, G. W., Morgan, W. P., Piering, P. N., Stegner, A. J., Stotesbery, C. L., Trine, M. R., \& Wu, M. Y. (1996). Psychological consequences of exercise deprivation in habitual exercisers. Medicine \& Science in Sports \& Exercise, 28(9), 1199-1203.

25. Ogden, J., Veale, D., \& Summers, Z. (1997). The development and validation of the Exercise Dependence Questionnaire. Addiction Re search \& Theory, 5(4), 343-355.

26. Olmedilla, A., Ortega, E., \& Gómez, J. M. (2014). Influencia de la lesión deportiva en los cambios del estado de ánimo y de la ansiedad precompetitiva en futbolistas. Cuadernos de Psicología del Deporte, $14(1), 55-62$.

27. Reche, C. \& Gómez, M., en prensa. Dependencia al ejercicio físico y trastornos de la conducta alimentaria. Apuntes de psicología.

28. Remor, E. (2005). Dependencia del ejercicio físico: ¿hecho o ficción? Psicologia Conductual, 13(3), 395-404.

29. Rosa, D. A., Mello, M. D., \& Souza-Formigoni, M. L. O. (2003). De pendência da prática de exercícios físicos: estudo com maratonistas brasileiros. Revista Brasileira de Medicina do Esporte, 9(1), 9-14.

30. Rosa, D. A., De Mello, M. T., Negrăo, A. B., \& De Souza-Formigoni, M. L. O. (2004). Mood changes after maximal exercise testing in subjects with symptoms of exercise dependence. Perceptual and motor skills, 99(1), 341-353.

31. Sicilia, A. \& González, D. (2011). Dependence and physical exercise Spanish Validation of the exercise dependence scale-revised (EDS-R). Spanish Journal of Psychology, 14 (1), 421-431.

32. Tello, G., Jouvion, A., Boulard, J. F., Marimoutou, C., Cazenave, N. \& Théfenne, L. (2012). Exercise dependence and injuries in the "Centre national d'entrainement commando". Science \& Sports, 27(5), 274282.

33. Terry, A., Szabo, A. \& Griffiths, M. (2004). The exercise addiction inventory: a new brief screening tool. Addiction Research and Theory, 12, 489-99.

34. Veale, D. M. W. (1987). Exercise dependence. British Journal of Addiction, 82, 735-40.

35. Veale, D. M. W. (1995). Does primary exercise dependence really exist? En: J. Annet; B. Cripps \& H. Steinberg (editors). Exercise addiction: Motivacion for participation in sport and exercise. Leicester, UK: The British Psychological Society.

36. Weik, M. \& Hale, B. D. (2009). Contrasting gender differences on two measures of exercise dependence. British Journal of Sports Medicine, 43(3), 204-207. 